\title{
The OTO Specimen Preparation Method for Optimal Scanning Electron Microscopy Imaging of Pseudomonas aeruginosa
}

\author{
Judy Tsz Ying Lee ${ }^{1}$, Nanthawan Avishai ${ }^{2}$, and Kwai Ping Connie Tam ${ }^{1,3}$ \\ 1. Departments of Ophthalmic Research, Cleveland Clinic Cole Eye Institute, Cleveland, OH, USA. \\ 2. Swagelok Center for Surface Analysis of Materials, Case Western Reserve University, Cleveland, $\mathrm{OH}$, \\ USA. \\ 3. Departments of Ophthalmic Research, Cleveland Clinic Lerner College of Medicine, Cleveland, $\mathrm{OH}$, \\ USA.
}

Pseudomonas aeruginosa, a ubiquitous gram-negative rod-shaped bacterium, has been intensively studied as an opportunistic human pathogen. It is one of the most common pathogens for nosocomial infection in immunocompromised individuals, e.g. cystic fibrosis patients [1]. Scanning electron microscopy (SEM) is a useful tool for obtaining detailed surface topography of microorganisms. For instance, it has been used for studying the ultrastructural basis of the resistance of $P$. aeruginosa to antiseptics, disinfectants and antibiotics [2]. Several methods for SEM sample preparation have been developed in order to enhance contrast, reduce structural damage and preserve cell structure in the native state. These techniques include glutaraldehyde fixation, negative staining, cryo-techniques, critical point drying, coating specimens with gold or osmium, and OTO staining. OTO staining has mostly been used in the preparation of biological tissues to provide bulk conductivity, which enables enhanced contrast [3]. Here, we report a specimen preparation protocol for optimal SEM imaging of $P$. aeruginosa using the OTO staining method.

In our optimized protocols, $P$. aeruginosa in the mid-exponential growth phase was washed and diluted with $0.9 \%$ sodium chloride to a cell density of $10^{8} \mathrm{cfu} / \mathrm{ml}$. It was then fixed with $2.5 \%$ glutaraldehyde in PBS and deposited onto a $0.4 \mu \mathrm{m}$-pore-size polycarbonate membrane. After fixation for 1 hour at room temperature and overnight at $4{ }^{\circ} \mathrm{C}$, it was post-fixed with $1 \% \mathrm{OsO}_{4}$ for 1 hour, followed by $1 \%$ thiocarbohydrazide (TCH) for $5 \mathrm{~min}$, and $1 \% \mathrm{OsO}_{4}$ again for $5 \mathrm{~min}$, with thorough washing between each step. The sample was dehydrated with graded ethanol series, and then impregnated with $50 \%$ hexamethyldisilazane (HMDS) in ethanol, and finally 100\% HMDS. The air-dried sample was sputtered with gold before imaging. As a comparison, we have also processed the sample without the steps of $1 \%$ TCH followed by $1 \% \mathrm{OsO}_{4}$.

Compared to the specimen prepared with the conventional method (Fig.1), the OTO method revealed significantly higher resolution ((Fig.2), which allows the observation of bacterial surface details. Our protocol also helps to preserve the cells during sample processing, as cell shrinkage was only observed in the conventional method. The improved image quality allows detection of subtle effects of any treatments to the bacterial surface morphology. 
References:

[1] J.M. Plotnikova, L.G. Rahme and F. M. Ausubel, Plant Physiol. 124 (2000), 1767-1774.

[2] U. Tattawasart, et al. J. of Antimicrobial Chemotherapy. 45 (2000), 145-152.

[3] S. V. Buravkov, V. P. Chernikov, and L. B. Buravkova. Bulletin of Experimental Biology and Medicine. 151 (2011), 378-382.

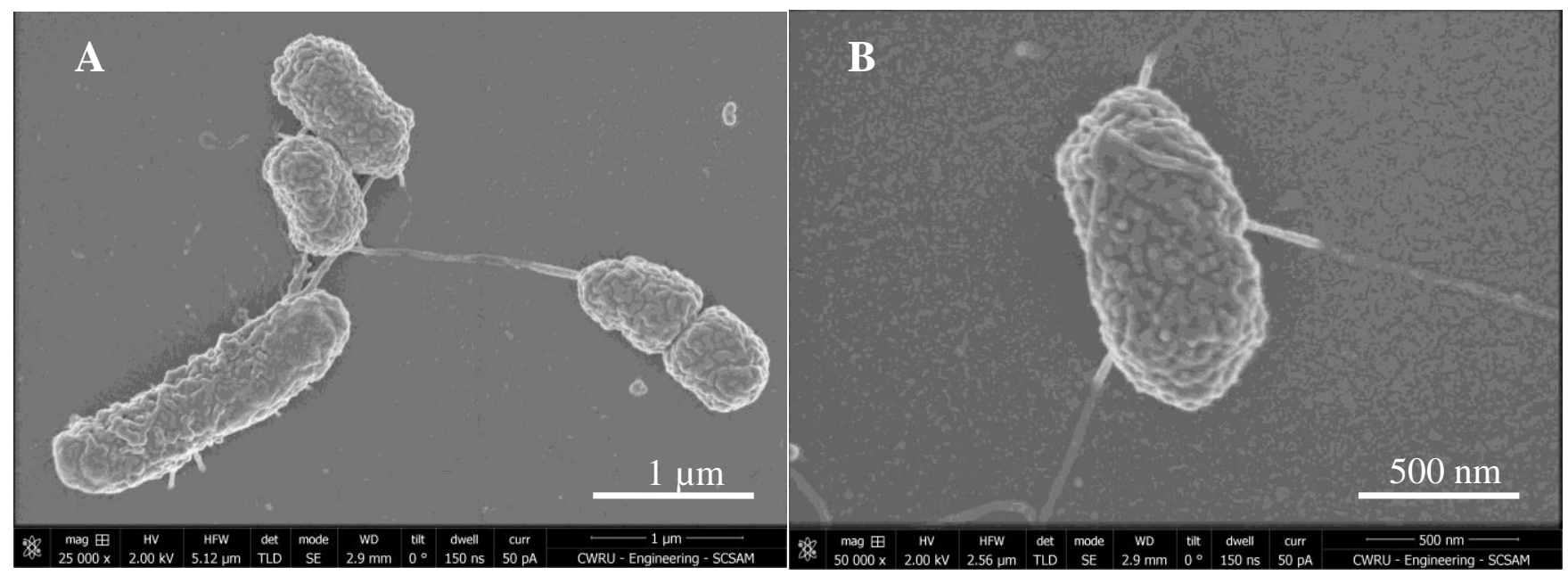

Figure 1. SEM images of $P$. aeruginosa using the conventional method for sample preparation at magnifications of 25,000x (A) and 50,000x (B). The SEM images were taken with FEI Helios Nanolab 650 using $2 \mathrm{KV}, 50 \mathrm{pA}$ with TLD mode 2 . Loss of surface components and cell shrinkage were observed.

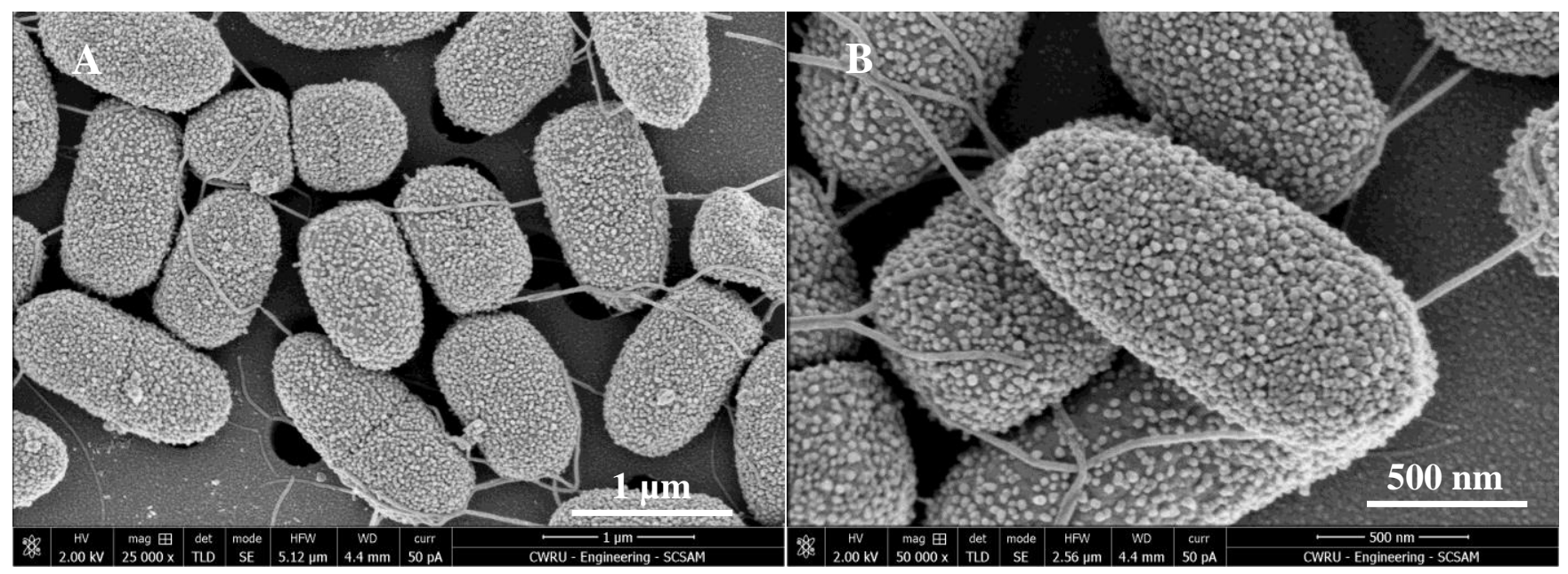

Figure 2. SEM images of $P$. aeruginosa using the OTO method for sample preparation at magnifications of 25,000x (A) and 50,000x (B). The SEM images were taken with FEI Helios Nanolab 650 using 2 KV, $50 \mathrm{pA}$ with TLD mode 2 . Bacterial surface components were well preserved and cell shrinkage was not observed. 